\title{
Fast Panoramic Face Mosaicing and Recognition
}

\author{
Fan Yang, Michel Paindavoine, Hervé Abdi* \\ Laboratoire Le2i, Aile de l'Ingénieur - Mirande \\ Université de Bourgogne, BP 400 - 21011 DIJON cedex, FRANCE \\ fanyang@u-bourgogne.fr, paindav@u-bourgogne.fr \\ *Program in Cognition and Neurosciences, MS: Gr.4.1 \\ The University of Texas at Dallas, Richardson TX 75083-0688 USA \\ herve@utdallas.edu
}

\begin{abstract}
In this article, we present some development results of a system that performs mosaicing (or mosaicking) of panoramic faces. Our objective is to study the feasibility of panoramic face construction in real-time. To do so, we built a simple acquisition system composed of 5 standard cameras which, together, can take simultaneously 5 views of a face at different angles. Then, we chose an easily hardware-achievable algorithm, consisting of successive linear transformations, in order to compose a panoramic face from these 5 views. The method has been tested on a relatively large number of faces. In order to validate our system of panoramic face mosaicing, we also conducted a preliminary study on panoramic faces recognition, based on the principal component method. Experimental results show the feasibility and viability of our system.
\end{abstract}

\section{Introduction}

Biometry is currently a very active area of research which comprises several sub-disciplines such as image processing, pattern recognition, and computer vision. The main goal of biometry is to build systems which can identify people from some observable characteristics such as their face, fingerprints, iris, etc. Faces seem to have a particularly strong appeal for the human users. Different techniques have been used to process faces[1][2][3]. As the recent DARPA-sponsored vendor test showed, most systems use frontal facial images as their input patterns[4]. As a consequence, most of these methods are sensitive to pose and lighting conditions. One way to override these limitations is to combine modalities (color, depth, 3D facial surface ... ) [5][6][7].

Most of 3D acquisition systems use professional devices such as a travelling camera or a 3D scanner [6][7]. Typically, these systems require that the subject remains immobile during several seconds in order to obtain a 3D scan, and therefore these systems may not be appropriate for some applications such as human expression categorization using movement estimation or real-time applications. Also, their cost can easily make these systems prohibitive for routine applications. In order to avoid using expensive and time intensive 3D acquisition devices, some face recognition systems generate 3D information from stereo-vision. Relatively complex calculations, however, are necessary in order to perform the auto-calibration and 2D projective transformation[8]. Another possible approach is to derive some 3D information from a set of face images, but without trying to reconstitute the complete $3 \mathrm{D}$ structure of the face[5][9].

The goal of the present article is to describe a system which is simple and efficient and also which can potentially process 3D faces in real-time. First, we describe our acquisition system. Then, we describe the method for creating panoramic face mosaics using successive linear transformations. Next, we present experimental results of panoramic face recognition. Finally we conclude and explore possible follow-ups and improvements.

\section{Acquisition system presentation}

Our acquisition system is composed of five Logitech 4000 USB cameras with a maximal resolution of $640 \times 480$ pixels. Each camera is fixed on a height-adjustable sliding support in order to adapt the camera position to each individual (see Figure 1). One of its main advantages is an easy setup and very low cost. The acquisition program grabs images from the 5 cameras simultaneously. These 5 images are stored in the PC with a frame data rate of $20 \times 5=100$ images per second. The human subject sits in front of the acquisition system, directly facing the central camera (Camera 3 ). Different color markers are placed on the subject's face. These markers are used later on to define common points between different face views. The position of these color markers corresponds roughly to the face fiduciary points. Figure 2 shows the position of the chosen points. There are 10 markers on each face with at least 3 markers in common between each face view. 



Figure 1. The Acquisition system: the left panel shows the 5 cameras and their support, the right panel shows the five images collected from a subject. Each image size is $240 \times 320$ pixels.

\section{Panoramic face construction}

Several panoramic image construction algorithms have been already introduced[9][10][11]. In general, the methods using non-linear transformations and iterative algorithms obtain very correct results in terms of geometric precision. However, these methods require a large number of computations and therefore cannot be easily implemented in real-time. Because we ultimately want to be able to build a real-time system, we decided to use simple (and therefore fast) linear methods. Our panoramic face construction algorithm is performed in three stages (see Figure 3):

1. Marker detection and marker coordinate calculation.

2. Transformation matrix estimation and image linear transformation.

3. Creation of panoramic face mosaics.

\subsection{Marker detection and marker coordinate cal- culation}

The first step of the algorithm corresponds to the detection of the markers put on the subject's face. We used 3 colors to create 10 markers (4 blue, 3 yellow, and 3 violet ones, see Figure 2 for an illustration). These markers were used as reference points for pasting the different views of the face. In order to detect the markers, we used color segmentation based upon the hue and saturation components of each image. This procedure allows a strong color selectivity and a small sensitivity to luminosity variation. Figure 4 illustrates the steps used for the yellow marker detection process. First, color segmentation gives, from the original image (Figure 4 top left), a binary image which contains the detected markers (Figure 4 top right). Then, in order to find the marker coordinates we used a logical AND operation which was performed between the binary image and a grid including white pixels separated by a fixed distance (Figure 4 bottom left). Finally, we computed the centers of the detected zones (Figure 4 bottom right). These centers gives the coordinates of the markers in the image.
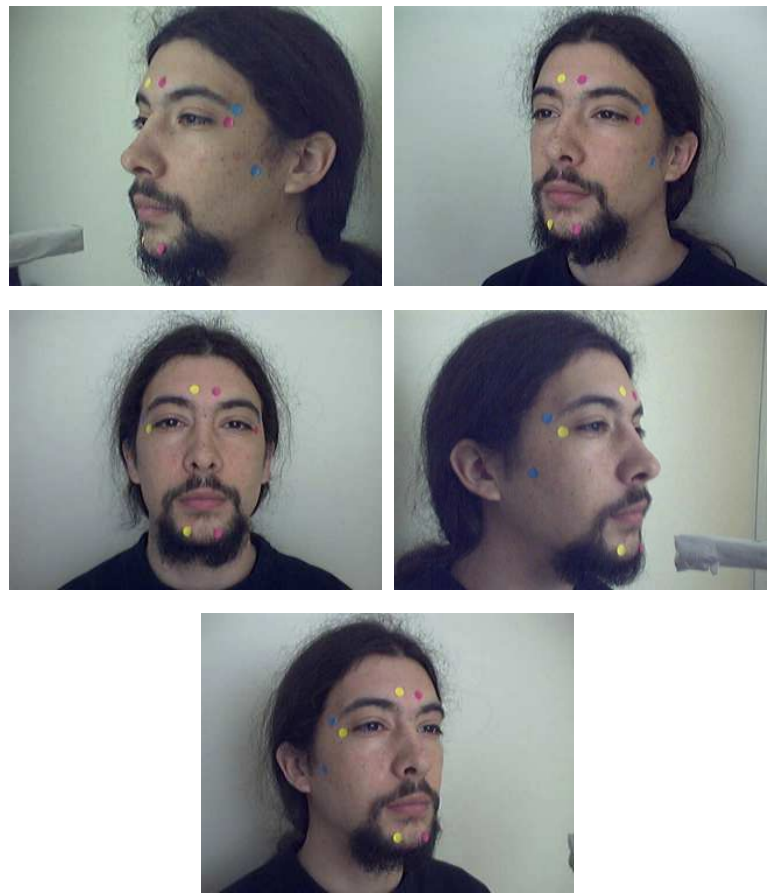

Figure 2. Distribution of 10 markers in 5 views (clockwise from top left): Image 1, Image 2, Image 3, Image 4, and Image 5.

\subsection{Transformation matrix estimation and image linear transformation}

We decided to represent each face as a mosaic which is made by concatenation of the different views pasted together as if they were on a flat surface. So, in order to create a panoramic face we combine the five different views. We start with the central view and paste the lateral views one at a time (see Figure 3). Our method consists of transforming the image to be pasted in order to link common points between this image and the target image. We obtain this transformed image by multiplying it by a linear transformation matrix. This matrix is calculated as a function of the coordinates of 3 common markers between 2 images. $\mathbf{C}_{1}$ and $\mathbf{C}_{2}$ represent, respectively, coordinates of the first and second images:

$$
\begin{aligned}
\mathbf{C}_{1} & =\left[\begin{array}{lll}
x_{1} & x_{2} & x_{3} \\
y_{1} & y_{2} & y_{3}
\end{array}\right] \\
\mathbf{C}_{2} & =\left[\begin{array}{lll}
x_{1}^{\prime} & x_{2}^{\prime} & x_{3}^{\prime} \\
y_{1}^{\prime} & y_{2}^{\prime} & y_{3}^{\prime}
\end{array}\right]
\end{aligned}
$$

We obtain the transformation matrix as follows:

$$
\mathbf{T}=\mathbf{C}_{1} \times\left(\mathbf{C}_{2}^{\star}\right)^{-1}
$$

with

$$
\mathbf{C}_{2}^{\star}=\left[\begin{array}{ccc}
x_{1}^{\prime} & x_{2}^{\prime} & x_{3}^{\prime} \\
y_{1}^{\prime} & y_{2}^{\prime} & y_{3}^{\prime} \\
1 & 1 & 1
\end{array}\right]
$$




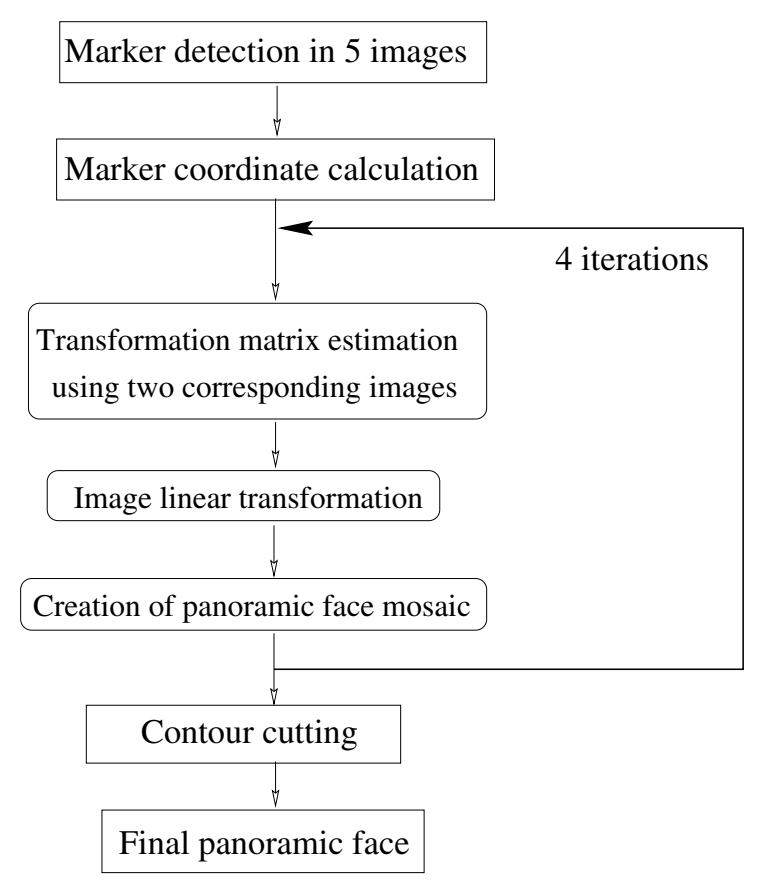

Figure 3. Block diagram of proposed panoramic face construction algorithm.

and

$$
\mathbf{T}=\left[\begin{array}{lll}
a_{1} & b_{1} & c_{1} \\
a_{2} & b_{2} & c_{2}
\end{array}\right]
$$

We generalize this transformation to the whole image :

$$
\begin{aligned}
& x=a_{1} x^{\prime}+b_{1} y^{\prime}+c_{1} \\
& y=a_{2} x^{\prime}+b_{2} y^{\prime}+c_{2}
\end{aligned}
$$

This linear transformation corresponds to a combination of image rotation, image translation, and image dilation (see Figure 5). Figure 6 displays the superposition of Image 3 (not transformed) and Image 4 (transformed using the coordinates of the yellow markers as common points).

\subsection{Creation of panoramic face mosaics}

We begin the panoramic face construction with the central view (Image 3, see Figure 2). From the superposition of the original Image 3 and transformed Image 4 (see Figure 6), we remove redundant pixels in order to obtain a temporary panoramic 3-4 image (see Figure 7 left). In order to eliminate redundant pixels, we create a cutting line which goes through two yellow markers. This panoramic 3-4 image temporarily becomes our target image.

We repeat this operation for each view. First, Image 2 is pasted on the temporary panoramic Image 3-4 in order to obtain a new temporary panoramic 2-3-4 image (see Figure 7 right). The corresponding transformation matrix is generated using three common violet markers. Then, we compute the transformation matrix which constructs Image 2-3-4-5 (see Figure 8 left) using two blue markers and one yellow marker (topmost). Finally, Image 1 is pasted to the
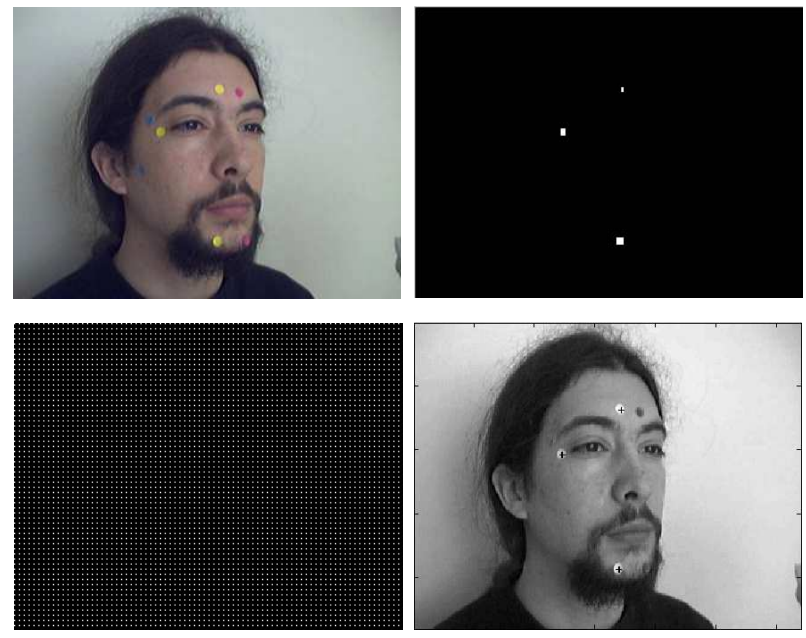

Figure 4. Yellow marker detection (reading left to right): original image, binary image after color filtering applied on Hue and Saturation components, a grid of white pixels, and marker coordinate localization.
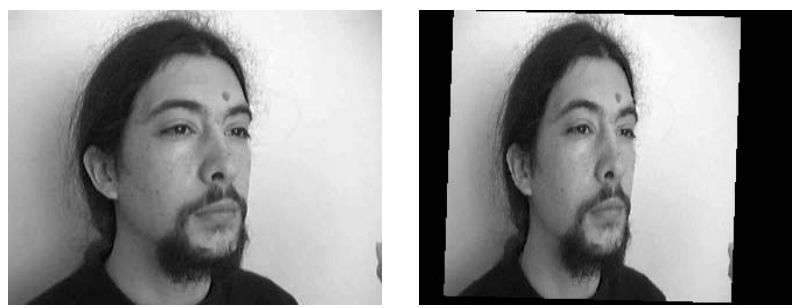

Figure 5 . Image 4 before and after the linear transformation: the transformation matrix is computed using Image 4 and Image 3 (central view, see Figures 2 and 6).


Figure 6. Superposition of Images 3 and 4: original Image 3 (left), and superposition of transformed Image $\mathbf{4}$ and original Image $\mathbf{3}$ (right).

temporary panoramic Image 2-3-4-5 with the help of two blue markers and one violet marker (topmost) (see Figure 8 right).

Figure 9 left displays the final panoramic face composition from 5 views. This composition preserves some of the face shape. For example, the chin in a human face possesses 

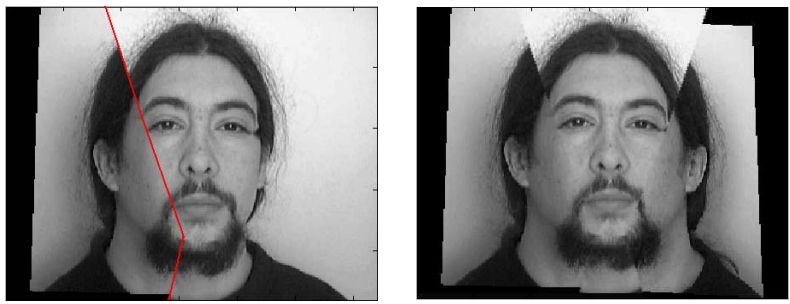

Figure 7. Mosaicing results: Image 3-4 (left), and Image 2-3-4 (right).


Figure 8. Mosaicing results: Image 2-3-4-5 (left), and Image 1-2-3-4-5 (right).
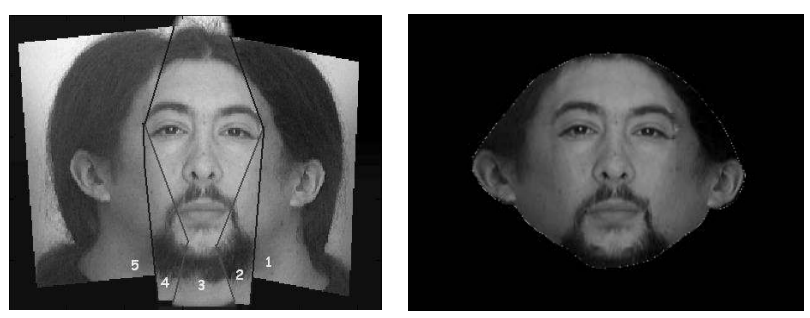

Figure 9. Mosaicing results: Panoramic face composition from 5 views (left), and final mosaic (right).

more curvature than other parts, therefore the bottom part of the panoramic face is composed of 5 views: 1, 2, 3, 4, and 5. On the other hand, 3 views $(1,3$, and 5) suffice to compose the top part.

Figure 9 right shows the final mosaic face obtained after automatically contour cutting. Note that these 10 markers allow us to link common points between 5 views. The coordinates of the markers are computed in the marker detection process, and arranged in a table. Then, all 10 markers are erased from all 5 views using a simple image processing technique (local smoothing).

We created a panoramic face database composed of 12 persons $\times 4$ expressions $\times 2$ sessions $=96$ panoramic faces. The two acquisition sessions were performed over an interval of one month. The four expressions were: neutral, smile, deepened eyebrows, and eyes closed (see Figure 10). We implemented a face recognition procedure using this database in order to test and validate the proposed panoramic face mosaicing algorithm.
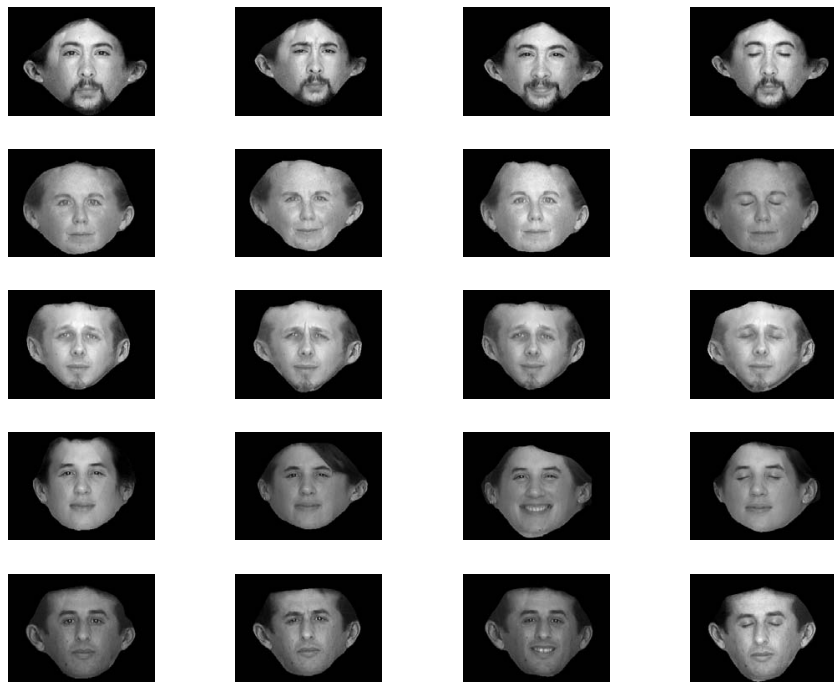

Figure 10. A sampling of panoramic faces from the first session's database.

\section{Face recognition description : PCA}

Over the past 25 years, several face recognition techniques have been proposed, One of the most versatile approach is derived from the statistical technique called Principal Component Analysis (PCA) adapted to face images[2][12][13]. PCA is based on the idea that face recognition can be accomplished with a small set of features that best approximates the set of known facial images.

Application of PCA for face recognition proceeds by first performing a PCA on a well-defined set of images of known human faces. From this analysis, a set of $K$ principal components is obtained and the projection of the new faces on these components is used to compute distances between new faces and old faces. These distances, in turn, are used to make predictions about the new faces. The complete set of patterns is represented by a $I \times K$ matrix noted A, where $I$ represents the number of pixels of the face images and $K$ the total number of images under consideration. Specifically, the learned matrix $\mathbf{A}$ can be expressed as:

$$
\mathbf{A}=\mathbf{P} \boldsymbol{\Delta} \mathbf{Q}^{T}
$$

where $\mathbf{P}$ is the matrix of eigenvectors of $\mathbf{A} \mathbf{A}^{T}, \mathbf{Q}$ is the matrix of eigenvectors of $\mathbf{A}^{T} \mathbf{A}$, and $\boldsymbol{\Delta}$ is the diagonal matrix of singular values of $\mathbf{A}, \boldsymbol{\Delta}=\boldsymbol{\Lambda}^{1 / 2}$ with $\boldsymbol{\Lambda}$ matrix of eigenvalues of $\mathbf{A} \mathbf{A}^{T}$ and $\mathbf{A}^{T} \mathbf{A}[13]$.

Given the singular vectors $\mathbf{P}$, every face in the database can be represented as a weight vector in the principal component space. The weights are obtained by projecting the face image onto the left singular vectors, and this is achieved by a simple inner product operation :

$$
\text { PROJ }_{x}=\mathbf{x}^{T} \mathbf{P} \boldsymbol{\Delta}^{-1}
$$

where $\mathbf{x}$ is a facial vector. It corresponds to an example face in the training process or a test face in the recognition process. 
Identification of the test image is done by locating the image in the known face database whose weights have the smallest Euclidean distance from the weight of the test image.

\section{Experimental results of panoramic face recognition}

\subsection{Spatial representation}

For these first tests, panoramic faces were analyzed using the original $240 \times 320$ pixel image (spatial representation) without pre-processing. The database consisted of 12 persons $\times 4$ expressions $\times 2$ sessions $=96$ panoramic faces, and was divided into two subsets. One subset served as the face training set and the other subset provided the face testing set. From the panoramic face database, one, two, or four images were randomly chosen for each individual in order to create the training set (number of patterns for learning per individual $p=1,2,4)$. The rest of the panoramic faces were used in order to test the face recognition method. Several executions of our Matlab program were run for each value of $p$ using randomly chosen training and testing sets. Then we computed mean performances, the results are presented in Table 1 where the recognition rate corresponds to correct panoramic face recognition rate. A discriminant analysis stage is added in the face recognition process in order to determine the number of necessary eigenvectors[14].

Table 1. Results of panoramic face recognition with spatial representation.

\begin{tabular}{|c|c|c|c|}
\hline $\begin{array}{c}\text { Number of training } \\
\text { examples/individual }\end{array}$ & $p=1$ & $p=2$ & $p=4$ \\
\hline $\begin{array}{c}\text { Number of total } \\
\text { training examples }\end{array}$ & 12 & 24 & 48 \\
\hline Number of eigenvectors & 9 & 13 & 25 \\
\hline $\begin{array}{c}\text { Number of tests } \\
\text { for recognition }\end{array}$ & 84 & 72 & 48 \\
\hline Recognition rate & $70 \%$ & $85.08 \%$ & $93.21 \%$ \\
\hline
\end{tabular}

\subsection{Frequential representation}

We also tested the frequential behaviour of our recognition system. We applied the same training and testing process for FFT amplitude signal of panoramic faces as used in spatial representation. Test results are given in Table 2. We obtain a better recognition rate with the frequential representation $(97.46 \%)$ than with the spatial representation (93.21\%).

This advantage of the frequential representation is due to the fact that for face images, the spectrum amplitude is less sensitive to noise (or variations) than the spectrum phase.
Table 2. Results of panoramic face recognition with frequential representation

\begin{tabular}{|c|c|c|c|}
\hline $\begin{array}{c}\text { Number of training } \\
\text { examples/individual }\end{array}$ & $p=1$ & $p=2$ & $p=4$ \\
\hline $\begin{array}{c}\text { Number of total } \\
\text { training examples }\end{array}$ & 12 & 24 & 48 \\
\hline Number of eigenvectors & 8 & 13 & 24 \\
\hline $\begin{array}{c}\text { Number of test } \\
\text { for recognition }\end{array}$ & 84 & 72 & 48 \\
\hline Recognition rate & $76.83 \%$ & $91.26 \%$ & $97.46 \%$ \\
\hline
\end{tabular}

We confirmed this interpretation by using a panoramic face image to which noise was added. Figure 11a shows a original panoramic face. Figure $11 \mathrm{~b}$ displays the same panoramic face image with added noise. We first performed the FFT of these two images and, then their inverse FFT in the two following manners:

1. Using the spectrum amplitude of the noised image and the spectrum phase of the original image (see Figure 11c).

2. Using the spectrum phase of the noised image and the spectrum amplitude of the original image (see Figure 11d).

These results show that the face obtained with the first configuration is closer to the original face than the face obtained with the second configuration. This confirms that the spectrum amplitude is less sensitive to noise than the spectrum phase.

\section{Conclusions and Perspectives}

In this article, we proposed a fast and simple method for panoramic face mosaicing. The acquisition system consists of several cameras followed by a series of fast linear transformations of the images. The simplicity of the computations makes it possible to envisage real-time applications.

In order to test the recognition performance of our system, we used the panoramic faces as input to a recognition system based upon principal component analysis. We tested two panoramic face representations: spatial and frequential. We found that a frequential representation gives the best performance with a correct recognition rate of $97.46 \%$ versus $93.21 \%$ for spatial representation.

Experimental results show that our fast mosaicing system provides relevant $3 \mathrm{D}$ facial surface information for recognition application. Obtained performance is very close or superior to published levels[1][5].

In the future, we plan to simplify our acquisition system by replacing the markers by a structured light. We also hope to use our system without markers. For this, we will detect control points on faces (corners and maximum curvature 



Figure 11. Amplitude is less sensitive to noise than phase (from left to right, top to bottom): a). An original panoramic face image; b). Original image with added Gaussian noise (mean $=0$ and variance $=0.05$ ); c). IFFT image using the spectrum amplitude of $b$ ) and the spectrum phase of a); and d). IFFT image using the spectrum amplitude of a) and the spectrum phase of $b$ ). The image c) is more similar to the image a) than the image d).

...). Another line of development is to improve the geometry quality of our panoramic face mosaic construction[9]. For this, we will use realistic human face models. We are also exploring processing panoramic face recognition using others classifiers with more variable conditions. Then, 3D face applications are envisaged such as real time human expression categorization using movement estimation and fast 3D facial modeling for compression and synthesis such as in video-conferencing.

\section{References}

[1] A.J. Howell and H. Buxton, Learning identity with radial basis function networks, Neurocomputing, Vol.20, pp.15-34, 1998.

[2] M. Turk and A. Pentland, Eigenfaces for recognition, Journal Cognitive neuroscience, Vol.3, pp.71-86, 1991.

[3] H. Abdi, D. Valentin and A. O'Toole, A generalized auto-associator model for face semantic process, in $O p$ timization and neural network, edited by D.Levine (Erlbaum, Hillsdale), 1997.

[4] P.J. Phillips, P. Grother and al., Face recognition Vendor Test 2002, IEEE International workshop on Analysis and Modeling of Faces and Gestures (AMFG), 2003.

[5] F. Tsalakanidou, D. Tzovaras and M.G. Strintzis, Use of depth and colour eigenfaces for face recognition, Pattern recognition Letters, Vol.24, pp.1427-1435, 2003.
[6] C. Hehser, A. Srivastava and G. Erlebacher, A novel technique for face recognition using range imaging, 7 th International Symposium on Signal Processing and its Applications (ISSPA), 2003.

[7] X. Lu, D. Colbry and A.K. Jain, Three-Dimensional model based face recognition, Proc. International Conference on Pattern Recognition, Cambridge, UK, August, 2004.

[8] R. Hartly and A. Zisserman, Multiple View Geometry in Computer vision, Cambridge University Press, Second Edition, 2003.

[9] X. Liu and T. Chen, Geometry-assisted statistical modeling for face mosaicing, IEEE International Conference on Image Processing (ICIP), Vol.2, pp.883-886, Barcelona, Spain, 2003.

[10] Y. Kanazawa and K. Kanatani, Image mosaicing by stratified matching, Image and Vision computing, Vol.22, pp.93-103, 2004.

[11] A.K. Jain and A. Ross, Fingerprint Mosaicing, IEEE International Conference on Acoustics, Speech, and Signal Processing (ICASSP), Orlando, Florida, May, 2002.

[12] D. Valentin, H. Abdi, A.J. O'Toole and G.W. Cottrell, Connectionist models offace processing: A survey, Pattern Recognition, Vol.27, 1208-1230, 1994.

[13] H. Abdi, A generalized approach for connectionist auto-associative memories: interpretation, implications and illustration for face processing. In J. Demongeot (Ed.), Artificial Intelligence and Cognitive Sciences. Manchester: Manchester University Press, (1988).

[14] A.J. O'Toole, F. Jiang, H. Abdi and J.V. Haxby, Partially distributed representations of objects and faces in ventral temporal cortex, Journal of Cognitive Neuroscience, Vol.17, (in press, 2005). 